Military Technical College

Kobry El-Kobba

Cairo, Egypt

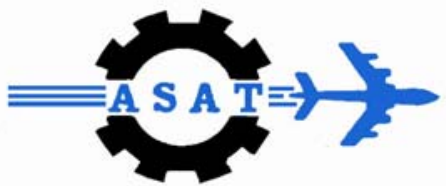

12-th International

Conference on

Aerospace Sciences \&

Aviation Technology

\title{
PERFORMANCE ANALYSIS OF MULTICARRIER FH/BFSK SYSTEM WITH JAMMING OVER RAYLEIGH FADING CHANNEL
}

\author{
EL-MAHDY *, A. E., ABOU-BAKR *, H.H., SAMY *, A.M.
}

\begin{abstract}
In this paper, the performance of a multicarrier frequency-hopping/binary frequencyshift keying (MC-FH/BFSK) system with jamming is evaluated. The communication channel is assumed to be Rayleigh fading channel. The considered types of jamming are partial band and broadband jamming. A closed-form expression of the average probability of error due to jamming is obtained. It is found that a smart jammer should be present in all subbands of the MC-FH/BFSK system in order to be effective. Also, a performance comparison between MC-FH/BFSK and single carrier FH/BFSK systems is presented. It is found that, the performance of MC-FH/BFSK under jamming is better than the performance of FH/BFSK.
\end{abstract}

\section{KEY WORDS}

Multicarrier systems, frequency hopping, fading, and jamming.

\footnotetext{
* Egyptian Armed Forces
} 


\section{INTRODUCTION}

The frequency-hopping $(\mathrm{FH})$ scheme has been primarily used to combat jamming signals in military communications environment. Two major types of jamming signals for an $\mathrm{FH}$ communication system are partial-band noise jamming (PBNJ) and multitone jamming (MTJ). The fast frequency-hopping binary frequency shift keying (FFH/BFSK) system using a product-combining receiver (PCR) provides good performance under narrowband partial-band jamming (PBJ) conditions [1]-[4]. The average bit error rate (BER) performance of FFH/BFSK PCR over a Rayleigh fading channel was discussed in [1] and [2]. The average BER expression for different orders of diversity is obtained. It is shown that an optimum diversity order exists under certain channel conditions. Other researchers extended the average BER of FFH/BFSK PCR to Rician and Nakagami fading channels [3], [4]. In the Rician fading channel, an $L$ number of integrators was used to derive the conditional BER, where $L$ is the diversity order. In [5] the BER performance of noncoherent FFH/BFSK noise normalization combining receiver is evaluated in the presence of PBJ and AWGN. The considered channels are independent frequency nonselective slowly Nakagami$\mathrm{m}$ fading channels. It is shown that a higher diversity level greatly improves the worst case performance of the receiver. The authors in [6] present the bit error rate performance of two suboptimum maximum- likelihood (ML) receivers for FFH/MFSK systems under the effect of multitone jamming (MTJ). The performance is derived for Rayleigh and Rician fading channel and validated by simulation. It is found that there exists an optimum diversity level for the two proposed suboptimum ML receivers. This diversity level is higher for the Rayleigh fading case than the Rician fading case. Other authors extended the analysis to differential FH system such as in [7] and [8]. In [7], the symbol error rate performance is analyzed over a Rayleigh fading channel with partial band noise jamming and AWGN. In [8], the performance of differentially $\mathrm{FH}$ with product combining receiver is studied and compared with other receivers.

As presented above, the authors do not focus in studying the performance of a MC$\mathrm{FH}$ system over a Rayleigh fading channel in presence of jamming. This system is recently used to overcome the problem of the fast $\mathrm{FH}$ systems which suffer from a significant performance loss due to using non-coherent demodulation and combining. In MC-FH systems, the frequency band is partitioned into $L$ disjoint subbands on which $L$ replicas of the signal are simultaneously transmitted. Since there is no hopping, coherent reception is easy to implement. Moreover, if side information on the jammer and noise level is available, optimum coherent combining results in a large improvement in error performance as compared to $\mathrm{FH}-\mathrm{SS}$ system.

In this paper, we study the performance of MC-FH/BFSK system in presence of partial band and broadband jamming over Rayleigh fading channel. The average error performance of this system is compared with that one of the FH/BFSK system under effect of jamming. The paper is organized as follows. The system model is described in section 2. The average bit error probability of the system under partial band jamming is derived in section 3. The performance of the system under broadband jamming is analyzed in section 4 . Numerical results are then given in section 5. Conclusions are provided in section 6. 


\section{SYSTEM MODEL}

The transmitter and receiver block diagrams of MC-FH system with coherent BFSK are shown in Fig.1, and Fig.2, respectively. The information symbols, $\left\{a_{k}\right\}$, are transmitted with a rate $1 / T_{b}, a_{k} \in\{0,+1\}$ and $k$ is the time index. The replicas of these bits are transmitted simultaneously over $L$ subbands. For simplicity, we assume that there is one frequency hop per signaling interval $T_{b}$. Let $W_{S S}$ be the total communicator bandwidth. Then the bandwidth of a subband, $W_{s b}$, is equal to $W_{S S} / L$. The bit $a_{k}$ is sent on $L$ branches, the replica of $a_{k}$ on branch $l ; l \in\{1, \ldots \ldots, L\}$ is denoted $a_{k, l}$. Each replica is transmitted using a BFSK modulation which occupies a frequency interval that is designated by subchannels. Obviously a subchannel is a subset of a subband. It is assumed that each subband contains $h=N / L$ different carrier frequencies spaced apart by $f_{d}$, where $N$ is the total number of carriers that can be accommodated in the communicator band. Thus the number of carriers in a subband is an integer $N / L$. It is clear that $h$ is the number of available hops for a symbol replica $a_{k, l} . f_{d}$ is chosen such that each pair of carriers is orthogonal i.e. $f_{d}=1 / T_{b}$, where $T_{b}$ is the bit time interval. The transmitted frequency hopped signal is given by

$$
s(t)=\sum_{k} \sum_{l=1}^{L} \sqrt{2 S} \exp \left[j 2 \pi\left(f_{l}+a_{k, l} f_{d}\right) t\right] P_{T_{b}}\left(t-k T_{b}\right)
$$

where $f_{l}$ is a carrier frequency in subband $l,\left\{a_{k, l}\right\}$ is binary sequence, and $P_{T_{b}}(t)$ is a unit rectangular pulse over interval $T_{b}$ which satisfies the orthogonality condition

$$
\int_{-\infty}^{\infty} P_{T_{b}}\left(t-m T_{b}\right) P_{T_{b}}\left(t-n T_{b}\right) d t=\delta_{m, n}\left\|P_{T_{b}}\right\|^{2} ; \text { where } \delta_{m, n}= \begin{cases}1 & \text { if } m=n \\ 0 & \text { otherwise }\end{cases}
$$

Note that, with the above assumptions, the hopper is different for each subband $l$. From (1), the total transmitted power is equal to $L S$ where $S$ is the transmitted power for one subband $l$. The frequency hopped signal at bit interval $k T_{b} \leq t \leq(k+1) T_{b}$ is given by

$$
s_{k}(t)=\sqrt{2 S} \exp \left(j 2 \pi\left(f_{l}+a_{k, l} f_{d}\right) t\right)
$$

The channel is assumed Rayleigh fading channel, the received signal is given by

$$
r(t)=g s(t)+n(t)
$$

where $g=\alpha \exp (j \theta)$ is a zero mean complex Gaussian random variable with a Rayleigh distributed amplitude $\alpha$ and a uniformly distributed phase angle $\theta$, and $n(t)$ is a sample function of a stationary zero mean complex Gaussian process, 
independent of signal and with psd $2 N_{0}$. The block diagram of the MC-FH/BFSK receiver is shown in Fig.1. To separate the signals of different carriers, $L$ branches are employed. Each branch contains a dehopper followed by two correlators. The dehopper of each branch knows the hopping pattern in that branch. The difference output of these correlators in each branch is multiplied by the conjugate of the fading parameter, $g_{l}^{*}$. The real part of the sum of outputs, $Z_{k, l}^{\prime}$, of all subbands gives the decision variable $Z_{k}$ which is then compared to zero threshold to make a decision about the information bit corresponding to that interval.

\section{PARTIAL BAND JAMMING}

The jamming signal, denoted $J(t)$, is a sample function of a stationary zero mean complex Gaussian process active on a fraction $\eta$ of the signal bandwidth. When the bandpass jammer is present on a hopping subchannel, we assume that it has a flat power spectral density $J_{0} / 2$. When the jammer is present in a subband, it covers a fraction $v$ of its bandwidth $W_{s b}$. Let $M$ be the number of subbands which jammed effectively. This means $M$ replicas of the data symbols are jammed in these subbands and $M \leq L$, then $\eta=v M / L$. If the jammer is present in all subbands then $M=L$ and $\eta=v$.

This section is divided into two subsections. In subsection $A$, a theoretical performance of the MC-FH/BFSK system in presence of partial band jamming in Rayleigh fading channel is derived. In subsection $B$, the performance of single carrier FH/BFSK under effect of partial band jamming is investigated for comparison purposes.

\section{A. Theoretical Performance Evaluation}

In this subsection, the performance of the MCFH/BFSK system is evaluated under partial band jamming. The performance is measured in terms of the bit error probability (BEP). Without loss of generality, we will consider the received signal in the interval corresponding to the zero bit. In the bit interval zero, the received signal is given by

$$
r(t)=\sum_{l=1}^{L} \sqrt{2 S} g_{l} \exp j 2 \pi\left(f_{l}+a_{0, l} f_{d}\right) t+n(t)+J(t)
$$

Consequently the output of the $\mathrm{i}^{\text {th }}$ dehopper, $q_{i}(t)$, is given by 


$$
\begin{aligned}
q_{i}(t)= & r(t) \exp \left(-j 2 \pi f_{i} t\right) \\
=\sum_{l=1}^{L} & \sqrt{2 S} g_{l} \exp j 2 \pi\left(f_{l}-f_{i}+a_{0, l} f_{d}\right) t+g_{l} n(t) \exp \left(-j 2 \pi f_{i} t\right) \\
& \quad+g_{l} J(t) \exp \left(-j 2 \pi f_{i} t\right)
\end{aligned}
$$

The outputs of the $i^{\text {th }}$ subband correlators, $Z_{0, i}$, is multiplied by the conjugate of the fading parameter, $g_{l}^{*}$, and the output, $Z_{0, i}^{\prime}$, is given by

$$
\begin{aligned}
Z_{0, i}^{\prime} & =\frac{1}{T_{b}} \int_{0}^{T_{b}} \sum_{l=1}^{L} \sqrt{2 S} \alpha_{l}^{2} \exp \left[j 2 \pi\left(f_{l}-f_{i}+a_{0, l} f_{d}-f_{d}\right) t\right] d t \\
& +\frac{1}{T_{b}} \int_{0}^{T_{b}} \alpha_{l}^{2} n(t) \exp \left[-j 2 \pi\left(f_{i}+f_{d}\right) t\right] d t+\frac{1}{T_{b}} \int_{0}^{T_{b}} \alpha_{l}^{2} J(t) \exp \left[-j 2 \pi\left(f_{i}+f_{d}\right) t\right] d t \\
& -\frac{1}{T_{b}} \int_{0}^{T_{b}} \sum_{l=1}^{L} \sqrt{2 S} \alpha_{l}^{2} \exp \left[j 2 \pi\left(f_{l}-f_{i}+a_{0, l} f_{d}\right) t\right] d t-\frac{1}{T_{b}} \int_{0}^{T_{b}} \alpha_{l}^{2} n(t) \exp \left(-j 2 \pi f_{i} t\right) d t \\
& -\frac{1}{T_{b}} \int_{0}^{T_{b}} \alpha_{l}^{2} J(t) \exp \left(-j 2 \pi f_{i} t\right) d t
\end{aligned}
$$

where $\alpha_{l}^{2}=g_{l} g_{l}^{*}$. Moreover, as the different carriers are orthogonal, the integral in the first term of (7) is nonzero only for $l=i$ and otherwise equal to zero. Assuming that the transmitted bit at the interval number zero, $a_{0,1}$, is equal to, 1 , where $1 \leq l \leq L$, and substituting by $\alpha_{l}^{2}$ in (7), consequently we have:

$$
Z_{0, i}^{\prime}=\alpha_{i}^{2} \sqrt{2 S}+n_{i}+J_{i}
$$

Where $n_{i}$ is the complex noise component at the $i^{\text {th }}$ subband correlator and it is given by:

$$
n_{i}=\frac{\alpha_{i}^{2}}{T_{b}}\left[\int_{0}^{T_{b}} n(t) \exp \left[-j 2 \pi\left(f_{i}+f_{d}\right) t\right] d t-\int_{0}^{T_{b}} n(t) \exp \left(-j 2 \pi f_{i} t\right) d t\right]
$$

and, $J_{i}$ is the complex jamming component at the ith subband correlator output and it is given by:

$$
J_{i}=\frac{\alpha_{i}^{2}}{T_{b}}\left[\int_{0}^{T_{b}} J(t) \exp \left[-j 2 \pi\left(f_{i}+f_{d}\right) t\right] d t-\int_{0}^{T_{b}} J(t) \exp \left(-j 2 \pi f_{i} t\right) d t\right]
$$

The jamming power appears as an additional additive Gaussian noise source in each of the $M$ jamming subbands with power spectral density $J_{0}=J_{a v} / \eta W_{s s}$, where $J_{a v}$ is 
the total average jamming power. Assume that $M$ out of $L$ replicas are hit by the jammer, then as described in section 2 , the decision variable $Z_{k}$ is equal to

$$
Z_{k}=\operatorname{Re}\left\{\sum_{i=1}^{L} Z_{0, i}^{\prime}\right\}=\sqrt{2 S} \sum_{i=1}^{L} \alpha_{i}^{2}+\sum_{i=1}^{L} \operatorname{Re}\left(n_{i}\right)+\sum_{i=1}^{M} \operatorname{Re}\left(J_{i}\right)
$$

The decision variable $Z_{k}$ can be written as

$$
Z_{k}=\sqrt{2 S} \sum_{i=1}^{L} \alpha_{i}^{2}+N+J
$$

where $N=\sum_{i=1}^{L} \operatorname{Re}\left(n_{i}\right)$, and $J=\sum_{i=1}^{M} \operatorname{Re}\left(J_{i}\right) \cdot \ln (12), N$ is the total noise component and $J$ is the total jamming component caused by the partial band jammer. To obtain the distribution of the random variable $Z_{k}$, it is necessary to obtain the distribution of both $N$ and $J$. Again, as the carriers are orthogonal, $n_{i} \stackrel{s}{ }$ are independent and identically distributed (i.i.d.) Gaussian random variables. Consequently, $N$ is a Gaussian random variable (r.v.) with zero mean and variance $\sigma_{N}^{2}=\frac{2 N_{0}}{T_{b}} \sum_{i=1}^{L} \alpha_{i}^{2}$. Similarly $J$ is a Gaussian r.v. with zero mean and variance $\sigma_{J}^{2}=\frac{2 J_{0}}{T_{b}} \sum_{i=1}^{M} \alpha_{i}^{2}$. Since the noise and the jamming are independent, the variance of their sum, $\sigma_{z}^{2}$, is equal to the sum of their variances and it is given by

$$
\sigma_{z}^{2}=\frac{1}{T_{b}}\left(2 N_{0} \sum_{i=1}^{L} \alpha_{i}^{2}+2 J_{0} \sum_{i=1}^{M} \alpha_{i}^{2}\right)
$$

The pdf of their sum $(N+J)$ is obtained from convolution of their pdf and it is given by

$$
f_{Z_{k} / 1}(z)=\frac{1}{\sqrt{2 \pi \sigma_{z}^{2}}} \exp \left(\frac{-1}{2 \sigma_{z}^{2}}\left(z-\sqrt{2 S} \sum_{i=1}^{L} \alpha_{i}^{2}\right)^{2}\right)
$$

Note that the real of the decision variable $Z_{k}$ is compared with zero threshold to decide between one and zero. Then the bit error probability (BEP) is equal to the probability that the real of the decision variable is less than zero given that the transmitted bit is one. Then the conditional BEP, $P\left(e \mid M, \alpha_{i}\right)$, under partial band jamming given the transmitted bit is one can be written as 


$$
P\left(e \mid M, \alpha_{i}\right)=\int_{-\infty}^{0} f_{Z / 1}(z) d z=Q\left(\sqrt{\frac{E_{b}^{\prime} \sum_{i=1}^{L} \alpha_{i}^{2}}{N_{0}+J_{0}\left(\sum_{i=1}^{M} \alpha_{i}^{2} / \sum_{i=1}^{L} \alpha_{i}^{2}\right)}}\right)
$$

where $E_{b}^{\prime}$ is the energy per bit for the MC-FH/BFSK system which is given by $E_{b}^{\prime}=S T_{b}$. It is clear that, when $M$ increases, the degradation in system performance increases. The probability that $M$ out of $L$ replicas are hit simultaneously by the jammer is given by

$$
P_{M}=\left(\begin{array}{l}
L \\
M
\end{array}\right) v^{M}(1-v)^{L-M}
$$

Therefore the conditional bit error probability, $P\left(e \mid \alpha_{i}\right)$, of the system is given by

$$
P\left(e \mid \alpha_{i}\right)=\sum_{M=0}^{L} P_{M} P\left(e / M, \alpha_{i}\right)=\sum_{M=0}^{L}\left(\begin{array}{l}
L \\
M
\end{array}\right) \eta^{M}(1-\eta)^{L-M} Q\left(\sqrt{\frac{E_{b}^{\prime} \sum_{i=1}^{L} \alpha_{i}^{2}}{N_{0}+J_{o}\left(\sum_{i=1}^{M} \alpha_{i}^{2} / \sum_{i=1}^{L} \alpha_{i}^{2}\right)}}\right)
$$

When $M<L$, there are $(L-M)$ subbands without jamming which implies that when $E_{b} / N_{0}$ tends to infinity, $P_{e}$ tends to zero independent of jamming power. This is because there exist $(L-M)$ replicas of $a_{k}$ are found in the unjammed subbands and detected correctly. Therefore, the effective partial band jamming must exist in all subbands, that is $M=L$ and consequently $\eta=v$. Knowing that $J_{0}=N_{j} / \eta$, where $N_{j}$ is the jammer power spectral density, then with the assumption that $N_{0}<<N_{j}$, $P\left(e \mid \alpha_{i}\right)$ becomes

$$
P\left(e \mid \alpha_{i}\right)=\eta^{L} Q\left(\sqrt{\frac{\eta E_{b}^{\prime} \sum_{i=1}^{L} \alpha_{i}^{2}}{N_{j}}}\right)
$$

It is noted that, according to the error probability given in (18), the jammer can choose its parameter $\eta$ so that the communicator BEP is maximized. Before obtaining the average BEP, let us define some notations. The signal to jamming power ratio of channel $l$ is defined as $\gamma_{i j}=\eta \alpha_{i}^{2} E_{b}^{\prime} / N_{j}$ which for the independent and identically distributed Rayleigh fading channels has an exponential pdf given by $P_{\gamma_{i j}}\left(\gamma_{i j}\right)=\frac{1}{\bar{\gamma}_{j}} \exp \left(-\gamma_{i j} / \bar{\gamma}_{j}\right)$. Where $\bar{\gamma}_{j}$ is the average signal to jamming power ratio per 
bit which is given by $\bar{\gamma}_{j}=\eta \Omega E_{b}^{\prime} / 2 N_{j}$. Then, the total signal to jamming ratio per bit, $\gamma_{t j}$, at the output of the receiver is given by

$$
\gamma_{t j}=\sum_{i=1}^{L} \gamma_{i j}=\frac{\eta E_{b}^{\prime}}{N_{j}} \sum_{i=1}^{L} \alpha_{i}^{2}
$$

The pdf of $\gamma_{t j}$ is a chi-square and it is given by $P_{\gamma_{i j}}\left(\gamma_{t j}\right)=\frac{1}{(L-1) ! \bar{\gamma}_{j}^{L}} \gamma_{t j}{ }^{L-1} \exp \left(-\gamma_{t j} / \bar{\gamma}_{j}\right)$. Then using (18) and (19), $P\left(e \mid \alpha_{i}\right)$ can be written as

$$
P\left(e \mid \gamma_{t j}\right)=\eta^{L} Q\left(\sqrt{\gamma_{t j}}\right)
$$

Recall that, our goal is to evaluate the performance of the system in terms of average BEP. For this purpose the conditional BEP in (20) has to be statistically averaged over the random parameter $\gamma_{t j}$. The average BEP is given by

$$
\bar{P}_{e}=\int_{0}^{\infty} P\left(e \mid \gamma_{t j}\right) P_{\gamma_{t j}}\left(\gamma_{t j}\right) d \gamma_{t j}=\frac{\eta^{L}}{(L-1) ! \bar{\gamma}_{j}^{L}} \int_{0}^{\infty} \gamma_{t j}{ }^{L-1} Q\left(\sqrt{\gamma_{t j}}\right) \exp \left(-\gamma_{t j} / \bar{\gamma}_{j}\right) d \gamma_{t j}
$$

which, after successive by parts integrations, can be written as

$$
\overline{P_{e}}=\eta^{L}\left(\frac{1-\mu_{j}}{2}\right)^{L} \sum_{l=0}^{L-1}\left(\begin{array}{c}
L-1+l \\
l
\end{array}\right)\left(\frac{1+\mu_{j}}{2}\right)^{l}
$$

where $\mu_{j}$ is given by $\mu_{j}=\sqrt{\frac{\bar{\gamma}_{j}}{1+\bar{\gamma}_{j}}}$.

\section{B. Performance of Single Carrier FH/BFSK Systems in Presence of Partial Band Jamming}

In this subsection, the performance of the single carrier FH/BFSK in presence of partial band jamming over Rayleigh fading channel is presented for comparison purposes. The average BEP of single carrier FH/BFSK in the presence of partial band jamming can be obtained by substituting $L=1$ in (22) which results in

$$
\overline{P_{e}}=\frac{\eta}{2}\left(1-\mu_{j}\right)
$$

The performance of single carrier FH/BFSK under partial band jamming (represented by (23)) is compared with the performance of MC-FH/BFSK (represented by (22)) in 
section 5. The purpose of this comparison is to show superiority of MC-FH/BFSK system over the single carrier system in presence of partial band jamming.

\section{BROADBAND JAMMING}

In this section, the performance of MC-FH/BFSK system in presence of broadband jamming is provided. This section is divided into two subsections. In subsection $A$, a theoretical performance of the MC-FH/BFSK system in presence of broadband jamming in Rayleigh fading channel is derived. In subsection $B$, the performance of single carrier FH/BFSK under effect of broadband jamming is investigated for comparison purposes.

\section{A. Theoretical Performance Evaluation}

In broadband jamming the jammer spreads his total power $J$ uniformly across the total bandwidth $W_{S S}$. In this case, all subbands are jammed i.e. $M=L$ and the probability, $P_{M}$, that $M=L$ replicas are hit simultaneously by the jammer is the same for all subbands and is equal to $P_{M}=1 / L$. Therefore, in terms of $N_{j}, P\left(e \mid \alpha_{i}\right)$, of the system is represented by:

$$
P\left(e \mid \alpha_{i}\right)=\sum_{M=0}^{L} P_{M} P\left(e / M, \alpha_{l}\right)=Q\left(\sqrt{\frac{E_{b}^{\prime} \sum_{i=1}^{L} \alpha_{i}^{2}}{N_{j}}}\right)
$$

Following similar procedure as in the case of partial band jamming, the average BEP under broad band jamming is given by

$$
\overline{P_{e}}=\left(\frac{1-\mu}{2}\right)^{L} \sum_{l=0}^{L-1}\left(\begin{array}{c}
L-1+l \\
l
\end{array}\right)\left(\frac{1+\mu}{2}\right)^{l}
$$

where $\mu$ is given by $\mu=\sqrt{\frac{\bar{\gamma}}{1+\bar{\gamma}}}$, and $\bar{\gamma}=\Omega E_{b}^{\prime} / 2 N_{j}$ is the average signal to jamming ratio per bit.

\section{B. Performance of Single Carrier FH/BFSK System in Presence of broadband jamming}

The performance of the single carrier FH/BFSK system in presence of broadband jamming can be obtained by substituting $L=1$ in (25) and it results in 


$$
\overline{P_{e}}=\frac{1}{2}(1-\mu)
$$

The performance of MC-FH/BFSK under broad band jamming (given by (25)) is compared with the performance of single carrier FH/BFSK (given by (26)) in section 5.

\section{NUMERICAL RESULTS}

The plot of average BEP for MC-FH/BFSK under the effect of partial band jamming is illustrated in Fig.3, and Fig. 4, (eq.22). The figures are plotted for different values of $\eta$, and with $L=4$ and 8 respectively. These figures show that at any value of $\eta$, the average BEP decreases as $\bar{\gamma}_{j}$ increases. For example in Fig.3, at $\eta=0.75$ and $\bar{\gamma}_{j}=1 \mathrm{~dB}$, the average BEP is about $10^{-2}$ while at the same value of $\eta$ and $\bar{\gamma}_{j}=9 \mathrm{~dB}$, the average BEP is about $10^{-4}$. From another hand these figures show the effect of the subband order, $L$, on the average BEP where it is illustrated that as $L$ increases the average BEP decreases. For example in Fig. 3, at $\eta=0.5, \bar{\gamma}_{j}=10 \mathrm{~dB}$, and $L=4$ the average BEP is about $0.8 \times 10^{-4}$, while from Fig. 4 , where $L=8$ the average BEP is about $10^{-8}$ under the same values of $\eta$ and $\bar{\gamma}_{j}$. The plot of $\overline{P_{e}}$ for MC-FH/BFSK and single carrier FH/BFSK under partial band jamming is shown in Fig.5, (eq.22, and eq.23). This figure is plotted for $\eta=0.75$, and $L=4$. This figure shows that under partial band jamming the MC-FH/BFSK system has superior performance than the single carrier system. At $\bar{\gamma}_{j}=10 \mathrm{~dB}$, the $\overline{P_{e}}$ for MC-FH/BFSK system is $0.8 \times 10^{-4}$, while in the single carrier FH/BFSK system is about $0.5 \times 10^{-1}$. The plot of average BEP, $\overline{P_{e}}$, for MC-FH/BFSK under broad band jamming is shown in Fig.6, (eq.25). This figure is plotted with $L=4$ and 8 . It is illustrated from figure that under broadband jamming as $L$ increases the average BEP decreases. For example at $\bar{\gamma}=5 \mathrm{~dB}$ the $\overline{P_{e}}$ at $L=4$ is about $0.4 \times 10^{-2}$ while at $L=8$ is about $0.7 \times 10^{-4}$. The plot of average BEP, $\overline{P_{e}}$, for MC-FH/BFSK and single carrier FH/BFSK under broad band jamming is shown in Fig.7, (eq.25, and eq.26). This figure is plotted for $L=4$. It is illustrated from figure that under broadband jamming the MC-FH/BPSK system has superior performance than the single carrier system. For example at $\bar{\gamma}=5 \mathrm{~dB}$ the $\overline{P_{e}}$ for $\mathrm{MC}$ $\mathrm{FH} / \mathrm{BFSK}$ system is about $0.4 \times 10^{-2}$ while in the single carrier is about $10^{-1}$.

\section{CONCLUSIONS}

The performance of MC-FH/BFSK system in presence of jamming over Rayleigh fading channel has been analyzed. A closed-form expression of the average probability of error of MC-FH/BFSK system due to jamming has been obtained. It has been found that a smart jammer should be present in all subbands of the $\mathrm{MC}$ - 
FH/BFSK system in order to be effective. Also, a performance comparison between MC-FH/BFSK and single carrier FH/BFSK system has been presented. It is shown that, the performance of $\mathrm{MC}-\mathrm{FH} / \mathrm{BFSK}$ under jamming is better than the performance of $\mathrm{FH} / \mathrm{BFSK}$.

\section{REFERENCES}

[1] K. C. Teh, A. C. Kot, and K. H. Li, "Partial-band jamming rejection of FFH/BFSK with product combining receiver over a Rayleigh-fading channel," IEEE Commun. Lett., vol. 1, no. 3, pp. 64-66, May 1997.

[2] G. Huo and M. S. Alouini, "Another look at the BER performance of FFH/BFSK with product combining over partial-band jammed Rayleigh-fading channels," IEEE Trans. Veh. Technol., vol. 50, no. 5, pp. 1203-1215, Sep. 2001.

[3] K. C. Teh, A. C. Kot, and K. H. Li, "Performance of FFH/BFSK product combining receiver over a Rician-fading channel with partial-band jamming," Electron. Lett., vol. 33, no. 11, pp. 935-937, May 1997.

[4] T. C. Lim, W. He, and K. H. Li, "Rejection of partial-band noise jamming with FFH/BFSK product combining receiver over Nakagami fading channel," Electron. Lett., vol. 34, no. 10, pp. 960-961, May 1998.

[5] Li-ping Zhu, Yan Yao, and Yi-Sheng Zhu, "Antijam performance of FFH/BFSK with noise normalization combining in a Nakagami-m fading channel with partial band interference," IEEE Commun. Lett., vol. 10, no. 6, pp. 429-431, June 2006.

[6] Y. Han and Kah C, "Performance Study of Suboptimum Maximum- Likelihood Receivers for FFH/MFSK Systems with Multitone Jamming over Fading Channels," IEEE Trans. Veh. Technol., vol. 54, no. 5, pp. 82 -90, Jan. 2005.

[7] Z. Chen, S. Li, and B. Dong, "Performance analysis of differential frequency hopping system with partial band noise jamming over Rayleigh fading channels," in Proc. IEEE Vehicular Technology Conf., pp1 -5, Sept. 2006.

[8] Z. Chen, S. Li, and B. Dong, "Partial band jamming rejection of differential frequency hopping system with product combining receiver over Rayleigh fading channel," in Proc. IEEE International Conf., vol. 12, pp. 5612 - 5616, June 2006. 


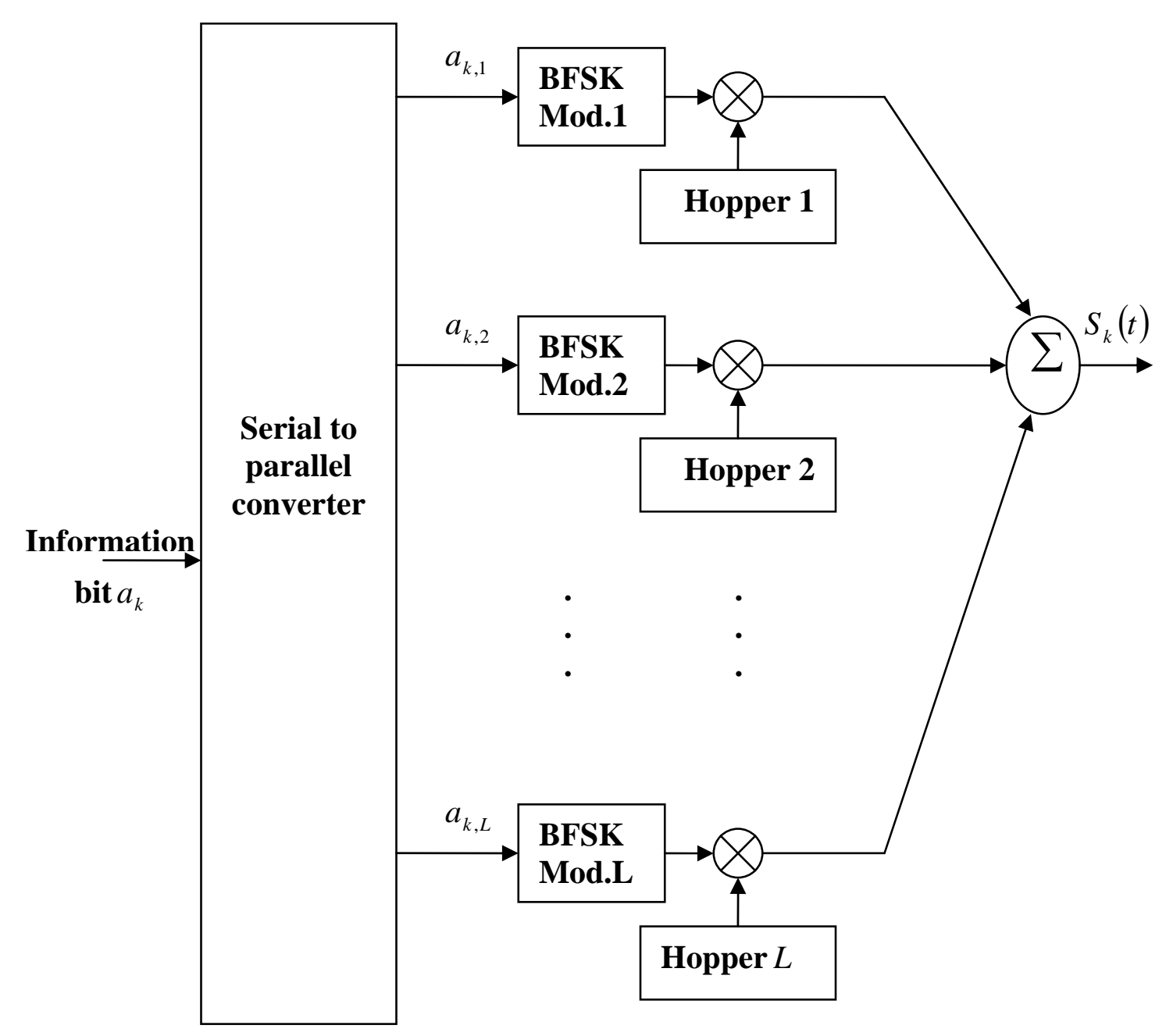

Fig.1. Block diagram of the MC-FH transmitter. 


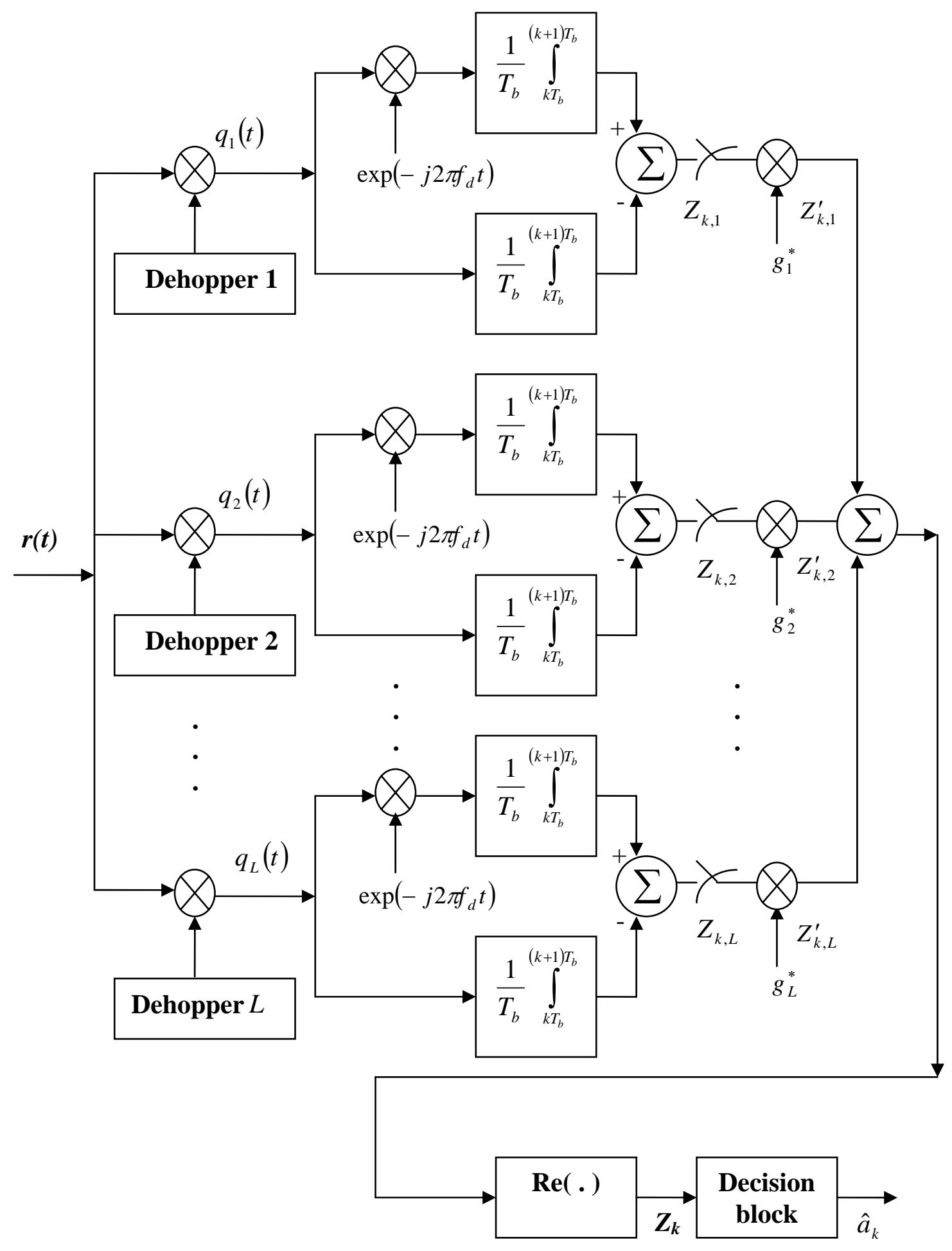

Fig.2. Block diagram of the MC-FH receiver. 


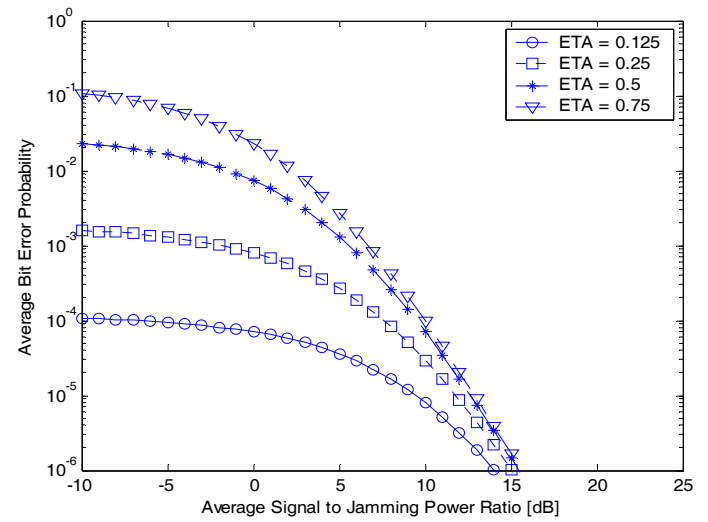

Fig.3.Average BEP of MC-FH/BFSK under partial band jamming at $L=4$.

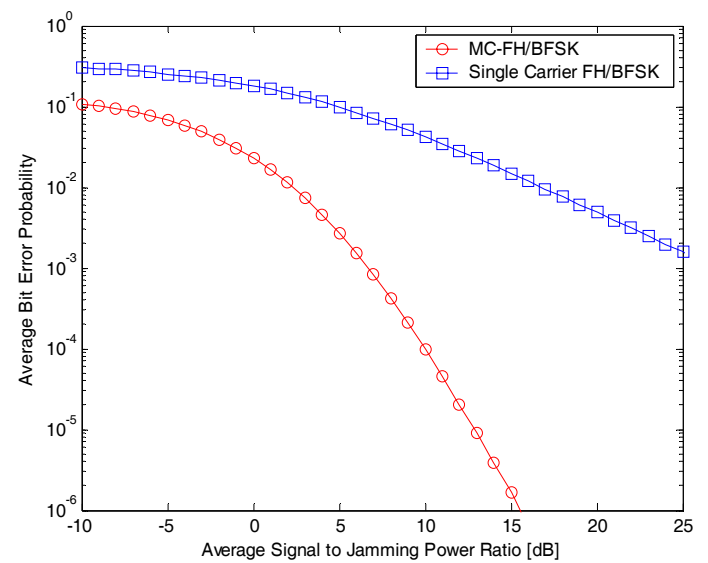

Fig.5. Performance comparison of single carrier FH/BFSK and MC-FH/BFSK under partial band jamming with $\eta=0.75$ and for $L=4$

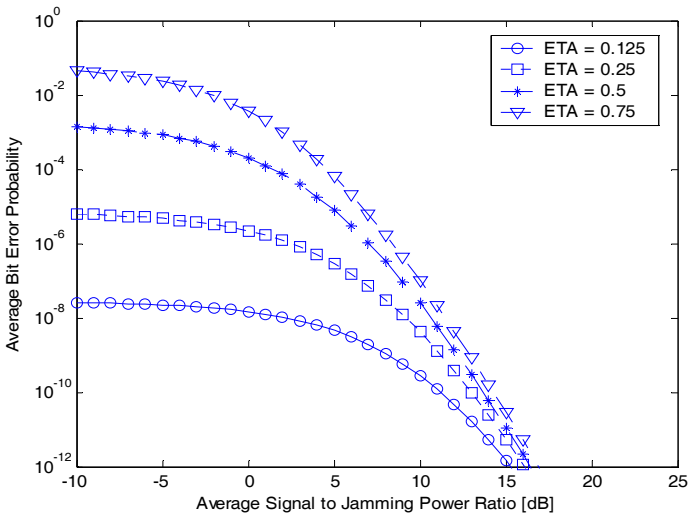

Fig.4. Average BEP of MC-FH/BFSK under partial band jamming at $L=8$.

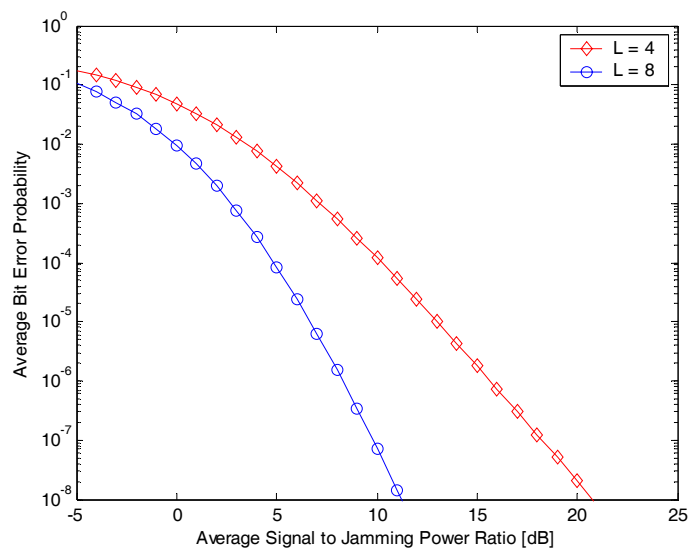

Fig.6. Average BEP under broad band jamming at $L=4$ and 8

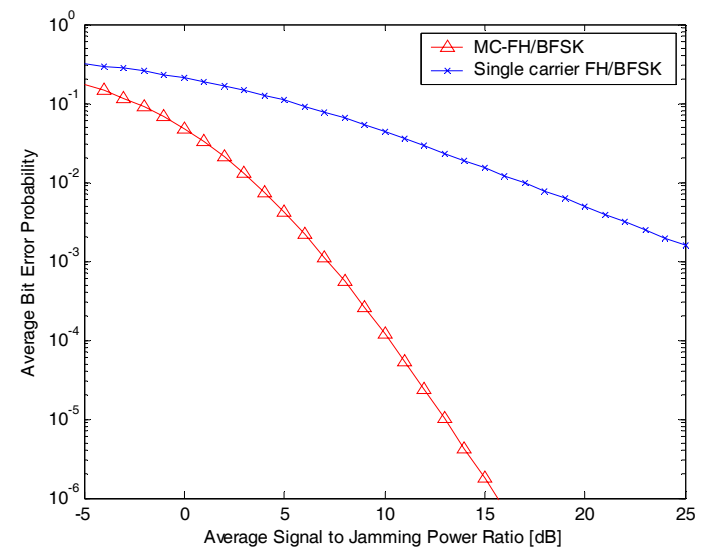

Fig.7. Performance comparison of single carrier FH/BFSK and MC-FH/BFSK under broadband jamming for $L=4$. 